\title{
SLIP IMPACT ON DIFFUSION OF A SOLUTE IN CREEPING SINUSOIDAL MOTION OF A NEWTONIAN FLUID WITH WALL FEATURES
}

\section{MALLINATH DHANGE* AND GURUNATH SANKAD}

\author{
B.L.D.E.A's V.P. Dr. P. G. Halakatti College of Engineering and Technology, Vijayapur, India \\ ${ }^{*}$ Corresponding author: math.mallinath@bldeacet.ac.in
}

\begin{abstract}
This article is concerned with the effect of slip boundary condition on two-dimensional creeping movement of a Newtonian fluid in the existence of pervious medium with wall features and heterogeneoushomogeneous chemical responses. The objective of this paper is to measure the performance of slip and wall feature constraints through graphs. It is observed that diffusion ascends with an increase in slip and wall constraints. The effective diffusion coefficient has been computed through long wavelength supposition and Taylor's condition for chemical responses.
\end{abstract}

\section{INTRODUCTION}

Mathematical modeling is the illustration of a system using scientific observation and linguistic. It is applied to study the complications in medical science. Bio-fluid dynamics is the branch of biomechanics which deals with the kinematics and dynamics of the fluids present in human beings, animals and plants. It spans from cells to organs, covering diverse aspects of functionality of systemic physiology, including cardiovascular, lymphatic, neurological, respiratory, reproductive, auditory and urinary systems. The biological systems are very complex and have defied all attempts at satisfactory mathematical solutions. These complicated systems are studied theoretically by means of approximated models whose simplified nature becomes amenable to mathematical analysis and give meaningful mathematical solutions. Hence the mathematical analysis and understanding of bio-fluid dynamics seem to be extremely important and useful for diagnosis and clinical

Received 2019-01-28; accepted 2019-03-14; published 2019-07-01.

2010 Mathematics Subject Classification. 76V05, 76Z05, 76S05, 92C10.

Key words and phrases. chemical reactions; creeping flow; diffusion; Newtonian fluid; wall features.

(C)2019 Authors retain the copyrights of their papers, and all open access articles are distributed under the terms of the Creative Commons Attribution License. 
purposes. The vital mechanism for fluid transportation in bio-fluid dynamics is Peristalsis. Peristalsis is a coordinated response wherein a wave of construction preceded by a wave of relaxation passes down a hollow viscus. From the perspective of fluid dynamics, peristalsis is typified by the dynamic interface of the fluid flow and movement of the flexible boundaries of the conduit. The study of the mechanism of peristaltic motion was first experimentally examined by Latham [1]. After this study, several experts have explored the creeping sinusoidal transportation of dissimilar liquids under various circumstances ( [2] - [4]). Mittra and Prasad [5] analyzed the movement of Newtonian liquid under peristalsis to know the effects of the viscoelastic behavior of walls. Further, a few investigators have studied the wall sound effects on different fluids with peristalsis ( [6] - [7]).

The dispersion is the process by which material is transported from one portion of a system to another as a result of random molecular motion. Dispersion of soluble matter in laminar flow has biological applications such as drug and nutrients distribution in the body. Through dispersion, metabolites are swapped between a cell and its environment or among the tissues and bloodstream. Due to its importance, many investigators explored the dispersion of a solute in Newtonian and non-Newtonian liquids under different limitations following Taylors approach( [8] - [12]).

Creeping stream with a pervious intermediate has attained significance in the current decade because of its practical applications chiefly in biomechanics and geophysical fluid dynamics. Even in some pathological situation like: transportation of liquid in kidneys, in lungs, gallbladder with stones, small blood vessels and tissues and bones and allocation of fatty cholesterol can be well thought-out as a pervious medium. The proper functioning of these depends on the stream of blood, nutrients, etc., through them. Hence, several authors studied influence of porosity in dissimilar liquids ( [13] - [19]). In many situations like physiological and engineering, the fluid slips at the walls of the channel. In slip conditions the boundary and the fluid moves with different velocity. It is important in describing the macroscopic effect of certain molecular phenomenon where interaction between fluid and solid occurs. The slip boundary condition was initially proposed by Beaver and Joseph [20]. Saffman [21] modified the periphery condition of Beaver and Joseph. The presence of slip phenomenon at the boundaries and interfaces has been observed in physiological streams, flows through pipes in which chemical responses take place at the walls. To the best of our knowledge, no attempt has yet been reported to discuss the impact of wall features and heterogeneous-homogeneous chemical responses on creeping flow of a Newtonian fluid in a pervious medium with a slip condition through Taylors approach.

\section{Mathematical Formulation and Methodology}

Consider the creeping flow of a Newtonian liquid through a pervious medium in the 2- dimensional conduit and assumed that conduit is packed with pervious material. Figure 1 displays the migrant waves. 


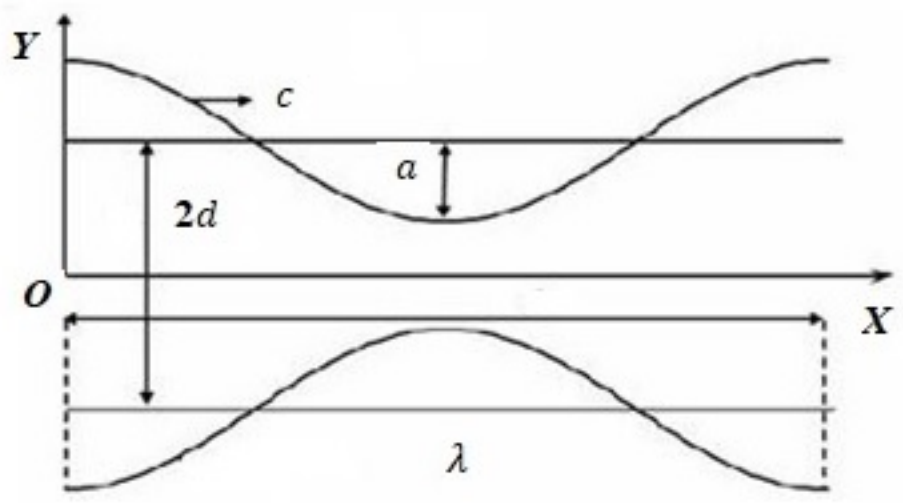

Figure 1. Physical model of the issu.

The migrant sinusoidal wave is given by the subsequent equation:

$$
\pm\left[a \sin \frac{2 \pi}{\lambda}(X-c t)+d\right]= \pm h=y
$$

The relating flow conditions of the present issue are:

$$
\begin{gathered}
0=\frac{\partial \mathcal{V}}{\partial y}+\frac{\partial \mathcal{U}}{\partial x} \\
-\frac{\partial p}{\partial x}+\mu \nabla^{2} \mathcal{U}-\frac{\mu}{\bar{k}} \mathcal{U}=\rho\left[\frac{\partial}{\partial t}+\mathcal{V} \frac{\partial}{\partial y}+\mathcal{U} \frac{\partial}{\partial x}\right] \mathcal{U} \\
-\frac{\partial p}{\partial y}+\mu \nabla^{2} \mathcal{v}-\frac{\mu}{\bar{k}} \mathcal{V}=\rho\left[\frac{\partial}{\partial t}+\mathcal{V} \frac{\partial}{\partial y}+\mathcal{U} \frac{\partial}{\partial x}\right] \nu,
\end{gathered}
$$

Referring [7], the condition of the springy wall movement is specified as:

$$
p-p_{0}=\mathcal{L}(h)
$$

where,

$$
-\mathcal{T} \frac{\partial^{2}}{\partial X^{2}}+m \frac{\partial^{2}}{\partial t^{2}}+\mathrm{C} \frac{\partial}{\partial t}=\mathcal{L} .
$$

Here, $\mathrm{C}$ - the coefficient of sticky damping force, $m$ - the mass per/area and $\mathcal{T}$ - the tension in the membrane.

Applying long wavelength hypothesis, conditions (2.2) to (2.4) yield as:

$$
\begin{gathered}
0=\frac{\partial \mathcal{V}}{\partial y}+\frac{\partial \mathcal{U}}{\partial x} \\
0=-\frac{\partial p}{\partial x}+\mu \frac{\partial^{2} \mathcal{U}}{\partial y^{2}}-\frac{\mu}{\bar{k}} \mathcal{U}
\end{gathered}
$$




$$
0=-\frac{\partial p}{\partial y}
$$

Following Bhatt and Sacheti [22], the allied border conditions are

$$
\mathcal{U}=-d \frac{\sqrt{D}_{a}}{\gamma} \frac{\partial \mathcal{U}}{\partial y} \text {, at } \quad y= \pm h .
$$

Where, $\gamma$ - slip constraint, $D_{a^{-}}$pervious constraint.

It is presumed that there is no horizontal displacement of the wall, $p_{0}=0$ and the channel walls are inextensible,

$$
\mu \frac{\partial^{2} \mathcal{U}}{\partial y^{2}}-\frac{\mu}{\bar{k}} \mathcal{U}=\frac{\partial}{\partial X} \mathcal{L}(h) \quad \text { at } \quad y= \pm h,
$$

where

$$
-\mathcal{T}^{\frac{\partial^{3} h}{\partial X^{3}}}+m \frac{\partial^{3} h}{\partial X \partial t^{2}}+\mathrm{C} \frac{\partial^{2} h}{\partial X \partial t}=\frac{\partial}{\partial X} \mathcal{L}(h)=\frac{\partial p}{\partial X} .
$$

Solving Eqns. (2.8), 2.9) with (2.10) and (2.11), we attain

$$
u(y)=\frac{1}{\mu m_{1}^{2}}\left(\frac{\partial p}{\partial x}\right)\left[A_{1}^{\prime} \cosh \left(m_{1} y\right)+A_{2}^{\prime} \cosh \left(m_{1} y\right)-1\right],
$$

The mean speed is specified and obtained as:

$$
\bar{u}(y)=\frac{1}{2 h} \int_{-h}^{h} \mathcal{u}(y) d y=\frac{1}{\mu m_{1}^{2}}\left(\frac{\partial p}{\partial x}\right)\left[\frac{A_{1}^{\prime}}{m_{1} h} \sinh \left(m_{1} h\right)-1\right] .
$$

Referring [13], the relative liquid speed is given as:

$$
\mathcal{U}_{x}=\mathcal{U}-\overline{\mathcal{U}}=\frac{1}{\mu m_{1}^{2}}\left(\frac{\partial p}{\partial x}\right)\left[A_{1}^{\prime} \cosh \left(m_{1} y\right)+A_{2}^{\prime} \sinh \left(m_{1} y\right)-A_{3}^{\prime}\right]
$$

where

$$
\begin{array}{r}
\mathcal{A}_{1}^{\prime}=\frac{1}{\left(1-\frac{D_{a}}{\gamma^{2}} d^{2} m_{1}^{2}\right) \cosh \left(m_{1} h\right)}, \quad \mathcal{A}_{2}^{\prime}=\frac{-\frac{\bar{D}_{a}}{\gamma} d m_{1}}{\left(1-\frac{D_{a}}{\gamma^{2}} d^{2} m_{1}^{2}\right) \cosh \left(m_{1} h\right)}, \quad \mathcal{A}_{3}^{\prime}=\left(\frac{\sinh \left(m_{1} h\right)}{\left(1-\frac{D_{a}}{\gamma^{2}} d^{2} m_{1}^{2}\right) m_{1} h \cosh \left(m_{1} h\right)},\right. \\
\mathcal{P}^{\prime}=-\mathcal{T} \frac{\partial^{3} h}{\partial X^{3}}+m \frac{\partial^{3} h}{\partial X \partial t^{2}}+\mathrm{C} \frac{\partial^{2} h}{\partial X \partial t}=\frac{\partial p}{\partial X}, \quad m_{1}=\sqrt{\frac{1}{k}} .
\end{array}
$$

Utilizing Taylor [8], the scattering equation for the concentration $\mathcal{C}$ of the material in isothermal circumstances is

$$
\mathcal{D} \frac{\partial^{2} \mathcal{C}}{\partial y^{2}}-k_{1} \mathcal{C}=U \frac{\partial \mathcal{C}}{\partial x}+\frac{\partial \mathcal{C}}{\partial t}
$$

Here, $k_{1}$ - the rate constant of first order chemical response, $\mathcal{D}$ - the molecular diffusion coefficient, and e - liquid concentration. 
It is expected that $\overline{\mathcal{U}} \approx \mathcal{e}([13])$, utilizing $\overline{\mathcal{U}} \approx \mathcal{e}$, and consequent non-dimensional quantities,

$$
\eta=\frac{y}{d}, \quad \theta=\frac{t}{\bar{t}}, \quad \bar{t}=\frac{\lambda}{\bar{u}}, \quad \mathcal{H}=\frac{h}{d}, \quad P=\frac{d^{2}}{\mu c \lambda} P^{\prime}, \quad \xi=\frac{(X-\bar{u} t)}{\lambda}, D_{a}=\frac{\bar{k}}{d^{2}} .
$$

Equations (2.15), (2.16) and (2.12) reduces to

$$
\begin{gathered}
\frac{d^{2}}{\mu m^{2}} \frac{\partial p}{\partial X}\left[\mathcal{A}_{1} \cosh (m \eta)+\mathcal{A}_{2} \cosh (m \eta)-\mathcal{A}_{3}\right]=\mathcal{U}_{x}, \\
\frac{\partial^{2} \mathcal{C}}{\partial \eta^{2}}-\frac{k_{1} d^{2}}{\mathcal{D}} \mathcal{C}=-\frac{d^{2}}{\lambda \mathcal{D}} \mathcal{U}_{x} \frac{\partial \mathcal{C}}{\partial \xi}, \\
-\epsilon\left[-\mathcal{E}_{3}(2 \pi)^{2} \sin (2 \pi \xi)+\left(\mathcal{E}_{1}+\mathcal{E}_{2}\right)(2 \pi)^{3} \cos (2 \pi \xi)\right]=\mathcal{P},
\end{gathered}
$$

where, $\mathcal{E}_{1}$-the rigidity $\mathcal{E}_{2}$-the stiffness, $\mathcal{E}_{3}$-the viscous damping force in the wall and $\epsilon$-the amplitude ratio.

The dispersion with 1st- order irreversible chemical response occur in the mass of the liquid and at the channel walls.

Referring [11], the wall conditions are specified as:

$$
\begin{gathered}
0=\mathcal{F}+\frac{\partial \mathcal{C}}{\partial y} \quad \text { at } \quad y=\left[a \sin \frac{2 \pi}{\lambda}(x-\bar{u} t)+d\right]=h, \\
0=-\mathcal{F C}+\frac{\partial \mathcal{C}}{\partial y} \quad \text { at } \quad y=-\left[a \sin \frac{2 \pi}{\lambda}(X-\bar{u} t)+d\right]=-h .
\end{gathered}
$$

After non-dimensionalisation, the Eqns. (2.21) and (2.22) yield as:

$$
\begin{gathered}
0=\beta \mathcal{C}+\frac{\partial \mathcal{C}}{\partial \eta} \quad \text { at } \quad \eta=[\epsilon \sin (2 \pi \xi)+1]=\mathcal{H}, \\
0=-\beta \mathcal{C}+\frac{\partial \mathcal{C}}{\partial \eta} \quad \text { at } \quad \eta=-[\epsilon \sin (2 \pi \xi)+1]=-\mathcal{H},
\end{gathered}
$$

where the heterogeneous response rate constraint is $\beta=\mathcal{F} d$, relating to catalytic response at the dividers.

Alluding Eqns. (2.23) and (2.24), the primitive of (2.19) is attained as:

$$
\mathcal{C}(\eta)=-\frac{d^{4}}{\lambda \mu \mathcal{D} m^{2}} \frac{\partial \mathcal{C}}{\partial \xi} \mathcal{P}\left[A_{4} \cosh (m \eta)+\mathcal{A}_{5} \sinh (m \eta)+\mathcal{A}_{6} \cosh (\alpha \eta)+\mathcal{A}_{7} \sinh (\alpha \eta)+\mathcal{A}_{8}\right]
$$

Here, $\alpha=\sqrt{\frac{k_{1}}{\mathcal{D}}} d, \quad m=m_{1} d=\sqrt{\frac{1}{D_{a}}}$.

The volumetric flow rate $\mathscr{Q}$ is specified and attained as:

$$
\mathscr{Q}=\int_{-\mathcal{H}}^{\mathcal{H}} \mathcal{C} \mathcal{U}_{x} d \eta=-2 \frac{d^{6}}{\lambda \mu^{2} \mathcal{D}} \frac{\partial \mathcal{C}}{\partial \xi} \mathcal{G}\left(\alpha, \beta, \epsilon, \mathcal{E}_{1}, \mathcal{E}_{2}, \mathcal{E}_{3}, \mathcal{D}_{a}, \gamma, \xi\right),
$$


where,

$$
\begin{aligned}
& \mathcal{G}\left(\alpha, \beta, \epsilon, \mathcal{E}_{1}, \mathcal{E}_{2}, \mathcal{E}_{3}, \mathcal{D}_{a}, \gamma, \xi\right)=\left\{\frac { \mathcal { P } ^ { 2 } } { m ^ { 4 } } \left[\frac{\mathcal{A}_{1} \mathcal{A}_{4}}{2} \mathcal{B}_{1}+\frac{\mathcal{A}_{1} \mathcal{A}_{5}}{2} \mathcal{B}_{2}+\left(\mathcal{A}_{1} \mathcal{A}_{8}-\mathcal{A}_{3} \mathcal{A}_{4}\right) \mathcal{B}_{3}-\mathcal{A}_{3} \mathcal{A}_{6} \mathcal{B}_{4}\right.\right. \\
& \left.\left.-\mathcal{B}_{5}+\mathcal{A}_{1} \mathcal{A}_{6} \mathcal{B}_{6}+\mathcal{A}_{2} \mathcal{A}_{7} \mathcal{B}_{7}\right]\right\}, \\
& \mathcal{A}_{1}=\frac{1}{\left(1-\frac{D_{a}}{\gamma^{2}} m^{2}\right) \cosh (m \mathcal{H})}, \quad \mathcal{A}_{2}=-\frac{\frac{\sqrt{\mathcal{D}}_{a}}{\gamma} m}{\left(1-\frac{D_{a}}{\gamma^{2}} m^{2}\right) \cosh (m \mathcal{H})}, \\
& \mathcal{A}_{3}=\frac{\sinh (m \mathcal{H})}{m \mathcal{H}\left(1-\frac{D_{a}}{\gamma^{2}} m^{2}\right) \cosh (m \mathcal{H})}, \mathcal{A}_{4}=\frac{1}{\left(m^{2}-\alpha^{2}\right)\left(1-\frac{D_{a}}{\gamma^{2}} m^{2}\right) \cosh (m \mathcal{H})}, \\
& \mathcal{A}_{5}=-\frac{\frac{\sqrt{\mathcal{D}}_{a}}{\gamma} m}{\left(m_{2}-\alpha^{2}\right)\left(1-\frac{D_{a}}{\gamma^{2}} m^{2}\right) \cosh (m \mathcal{H})}, \\
& \mathcal{A}_{6}=\frac{1}{\left(1-\frac{D_{a}}{\gamma^{2}} m^{2}\right) \cosh (m \mathcal{H})(\alpha \sinh (\alpha \mathcal{H})+\beta \cosh (\alpha \mathcal{H}))}\left\{\frac{-(m \sinh (m \mathcal{H})+\beta \cosh (m \mathcal{H}))}{\left(m^{2}-\alpha^{2}\right)}\right. \\
& \left.+\frac{\beta \sinh (m \mathcal{H})}{m \mathcal{H} \alpha^{2}}\right\} \\
& \mathcal{A}_{7}=\frac{\frac{\sqrt{\mathcal{D}}_{a}}{\gamma} m}{\left(1-\frac{D_{a}}{\gamma^{2}} m^{2}\right) \cosh (m \mathcal{H})} \frac{(m \cosh (m \mathcal{H})+\beta \sinh (m \mathcal{H}))}{(\alpha \cosh (\alpha \mathcal{H})+\beta \sinh (\alpha \mathcal{H}))} \\
& A_{8}=\frac{\sinh (m \mathcal{H})}{m \mathcal{H} \alpha^{2}\left(1-\frac{D_{a}}{\gamma^{2}} m^{2}\right) \cosh (m \mathcal{H})}, \\
& \mathcal{B}_{1}=\frac{\sinh (2 m \mathcal{H})+2 m \mathcal{H}}{2 m}, \quad \mathcal{B}_{2}=\frac{\sinh (2 m \mathcal{H})-2 m \mathcal{H}}{2 m}, \quad \mathcal{B}_{3}=\frac{\sinh (m \mathcal{H})}{m}, \quad B_{4}=\frac{\sinh (\alpha \mathcal{H})}{\alpha}, \\
& \mathcal{B}_{5}=\mathcal{A}_{3} \mathcal{A}_{8} \mathcal{H}, \\
& \mathcal{B}_{6}=\frac{m \sinh (m \mathcal{H}) \cosh (\alpha \mathcal{H})-\alpha \cosh (m \mathcal{H}) \sinh (\alpha \mathcal{H})}{m^{2}-\alpha^{2}}, \\
& \mathcal{B}_{7}=\frac{m \cosh (m \mathcal{H}) \sinh (\alpha \mathcal{H})-\alpha \sinh (m \mathcal{H}) \cosh (\alpha \mathcal{H})}{m^{2}-\alpha^{2}} .
\end{aligned}
$$

Looking at condition (2.27) with Fick's law of scattering, the dispersing coefficient $D^{*}$ was computed to such an extent that the solute disperses near to the plane moving with the typical speed of the flow and is specified as

$$
2 \frac{d^{6}}{\mu^{2} \mathcal{D}} \mathcal{G}\left(\alpha, \beta, \epsilon, \mathcal{E}_{1}, \mathcal{E}_{2}, \mathcal{E}_{3}, \mathcal{D}_{a}, \gamma, \xi\right)=\mathcal{D}^{*}
$$

Let $\overline{\mathcal{G}}$ be the average of $\mathcal{G}$, and is obtained by the following equation:

$$
\int_{0}^{1} \mathcal{G}\left(\alpha, \beta, \epsilon, \mathcal{E}_{1}, \mathcal{E}_{2}, \mathcal{E}_{3}, \mathcal{D}_{a}, \gamma, \xi\right) d \xi=\overline{\mathcal{G}}
$$




\section{Discussion of Outcomes}

The impact of various constraints on the mean effective scattering coefficient can be observed through the expression $\overline{\mathscr{G}}\left(\alpha, \beta, \epsilon, \mathcal{E}_{1}, \mathcal{E}_{2}, \mathcal{E}_{3}, \mathcal{D}_{a}, \gamma, \xi\right)$. Using MATHEMATICA software the graphs are plotted for Eqn. $(2.29)$.

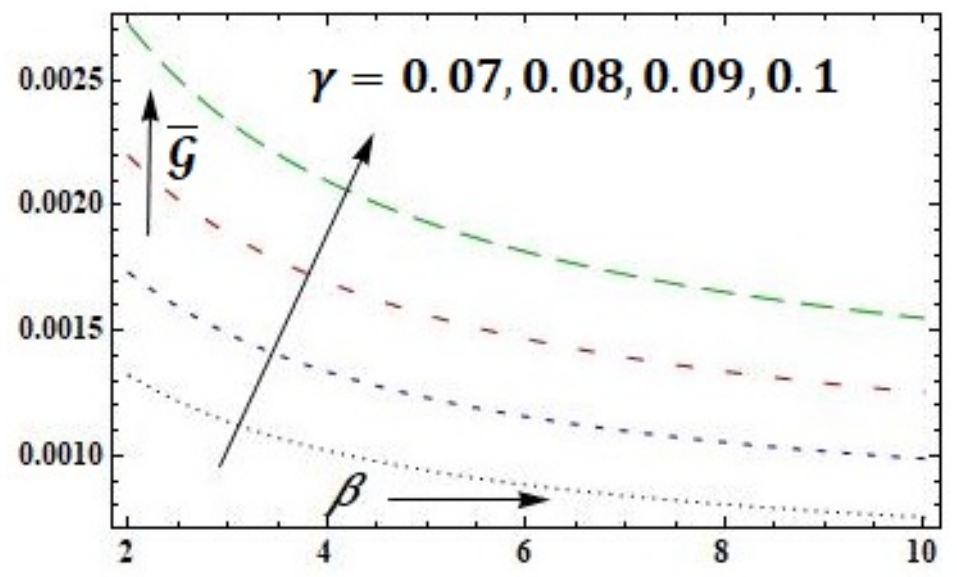

Figure 2. Illustration of $\gamma$ for $\overline{\mathcal{G}}$ at $\mathcal{E}_{1}=0.1, \mathcal{E}_{2}=0.0, \mathcal{E}_{3}=0.06, \epsilon=0.2, \alpha=1.0, \mathcal{D}_{a}=0.9$

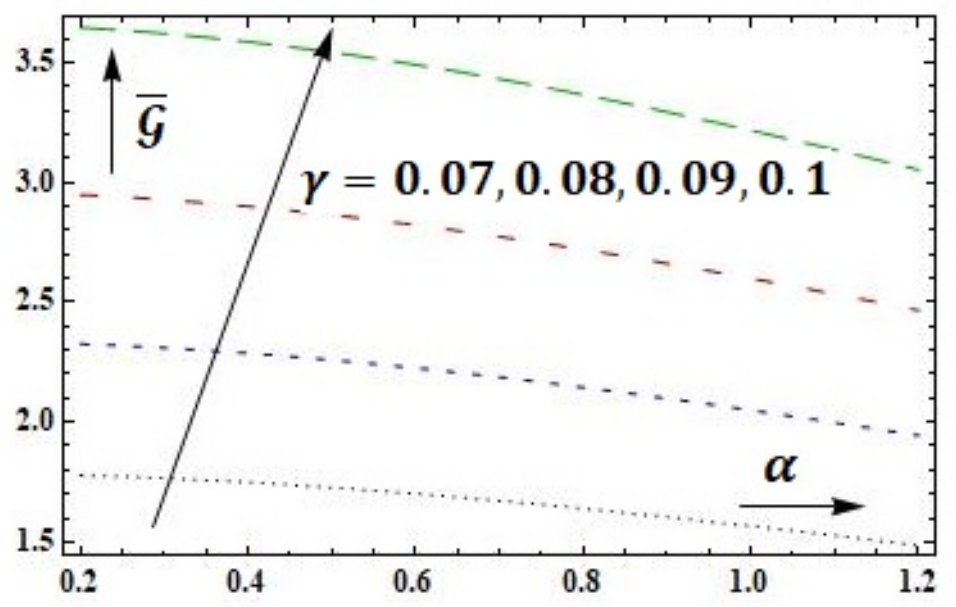

Figure 3. Illustration of $\gamma$ for $\overline{\mathcal{G}}$ at $\mathcal{E}_{1}=0.1, \mathcal{E}_{2}=4.0, \mathcal{E}_{3}=0.06, \epsilon=0.2, \beta=5.0, \mathcal{D}_{a}=0.9$

The effects of slip constraint on $\overline{\mathcal{G}}$ have been illustrated through the figures 2 - 4. It is noticed that, scattering rises as slip constraint $(\gamma)$ augments. As we already known that, heterogeneous response takes place more at the boundary of the conduit, due to slip boundary condition less heterogeneous response takes place at the boundary. Hence the dispersion is more in the case of no-slip boundary as compared to slip 


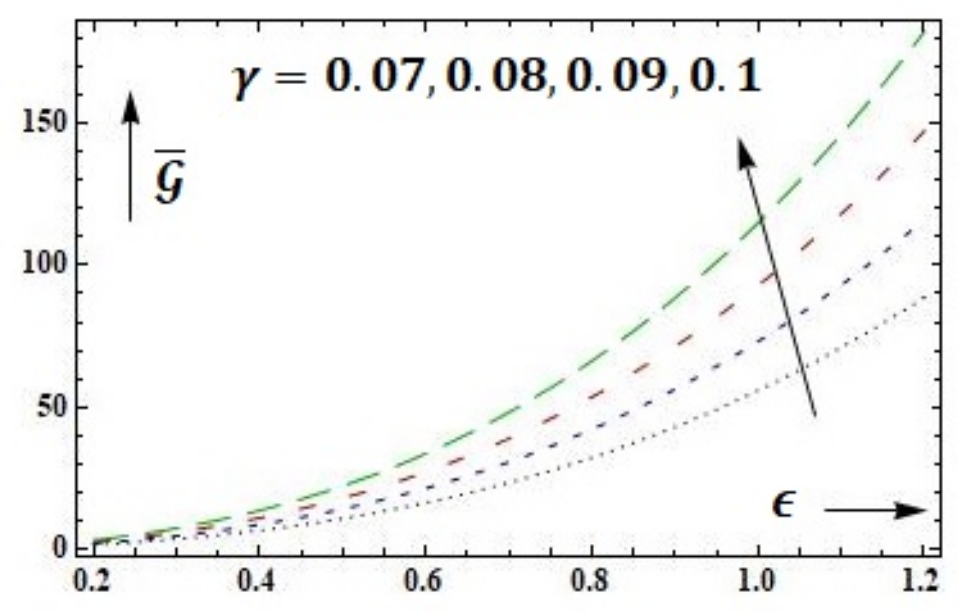

Figure 4. Illustration of $\gamma$ for $\overline{\mathcal{G}}$ at $\mathcal{E}_{1}=0.1, \mathcal{E}_{2}=4.0, \mathcal{E}_{3}=0.00, \alpha=1.0, \beta=5.0, \mathcal{D}_{a}=0.9$

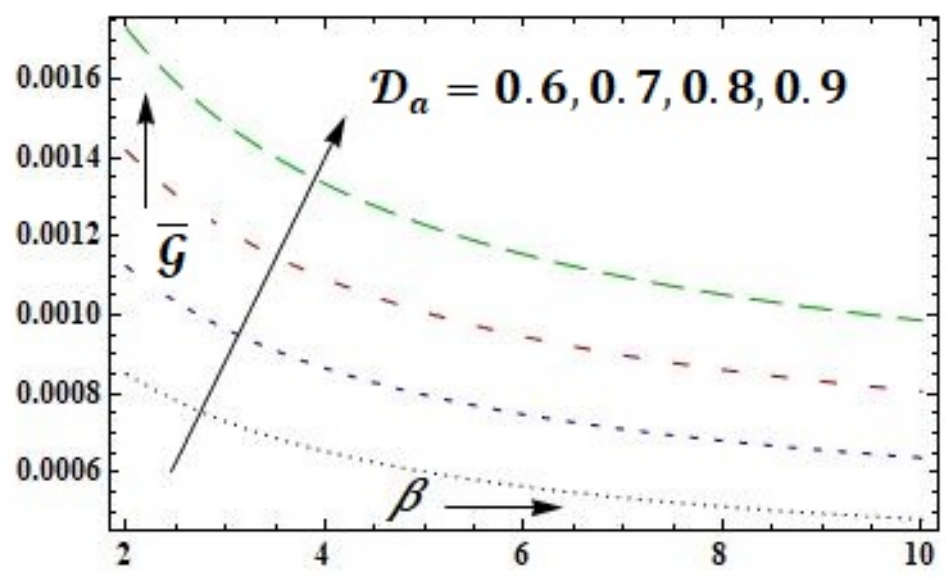

FiguRE 5. Illustration of $\mathcal{D}_{a}$ for $\overline{\mathcal{G}}$ at $\mathcal{E}_{1}=0.1, \mathcal{E}_{2}=0.0, \mathcal{E}_{3}=0.06, \epsilon=0.2, \alpha=1.0, \gamma=0.08$

boundary.

Figures $5-7$ indicates that $\overline{\mathcal{G}}$ enhances with a growth in the Darcy number $\left(D_{a}\right)$. This result concurs with the outcome of [6], [13], and [14].

Consider the figures 8 - 16 for the effect of the rigidity $\left(\mathcal{E}_{1}\right)$, stiffness $\left(\mathcal{E}_{2}\right)$ and viscous damping force $\left(\mathcal{E}_{3}\right)$ of the wall on the dispersal coefficient $(\overline{\mathcal{G}})$. It is observed that $\overline{\mathcal{G}}$ ascends monotonically with an increase in $\varepsilon_{1}, \varepsilon_{2}$ and $\varepsilon_{3}$. 


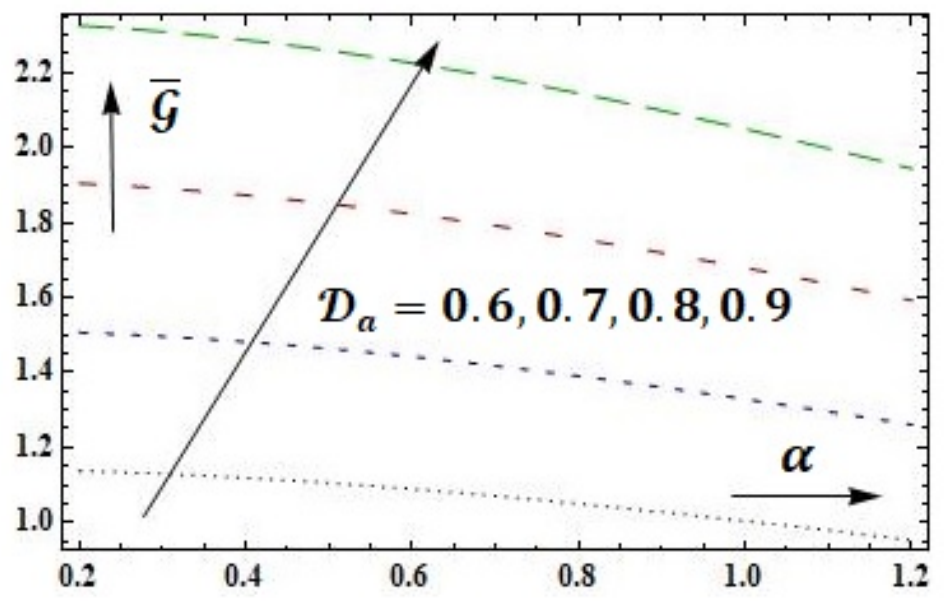

FiguRE 6. Illustration of $\mathcal{D}_{a}$ for $\overline{\mathcal{G}}$ at $\mathcal{E}_{1}=0.1, \mathcal{E}_{2}=4.0, \mathcal{E}_{3}=0.06, \epsilon=0.2, \beta=5.0, \gamma=0.08$

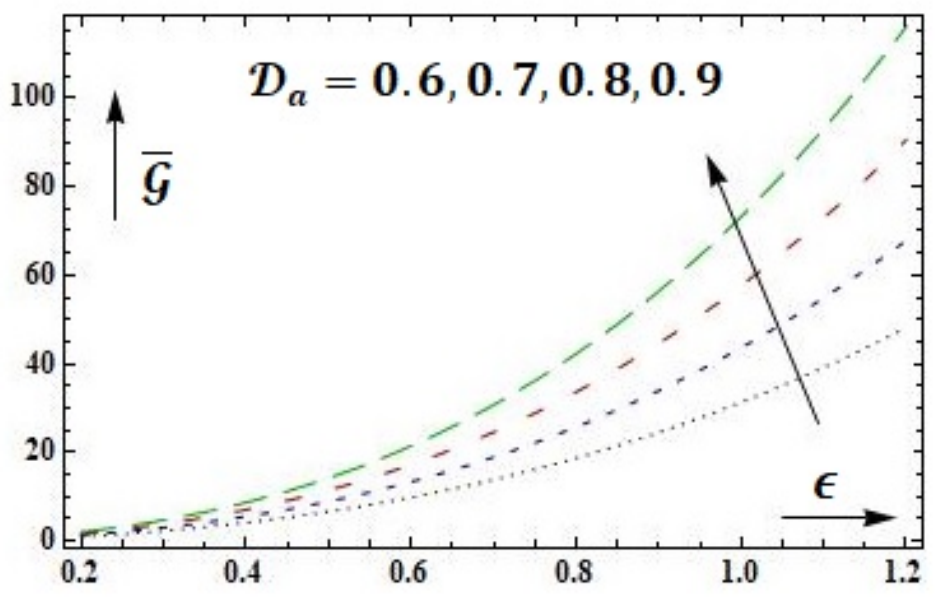

Figure 7. Illustration of $\mathcal{D}_{a}$ for $\overline{\mathcal{G}}$ at $\mathcal{E}_{1}=0.1, \mathcal{E}_{2}=4.0, \mathcal{E}_{3}=0.00, \alpha=1.0, \beta=5.0, \gamma=0.08$

Wall feature affects the increase in velocity of the liquid in conduit which reasons to enhance the scattering. These outcomes agree with the results of [7]. Figures 2, 5, 8, 11, 14 display that scattering lessens with heterogeneous substance response rate $\beta$ and also figures $3,6,9,12$, and 15 clear that scattering descends as homogeneous compound response rate $\alpha$ falls down.

It is observed that the solution expression for $\overline{\mathcal{G}}$ be in agreement with Sobh [15] when there is no wall features. Further, it is noticed that $\overline{\mathcal{G}}$ be in agreement with that [13] and [17], if there is absence of slip boundary condition ( i.e. with no-slip boundary condition). 


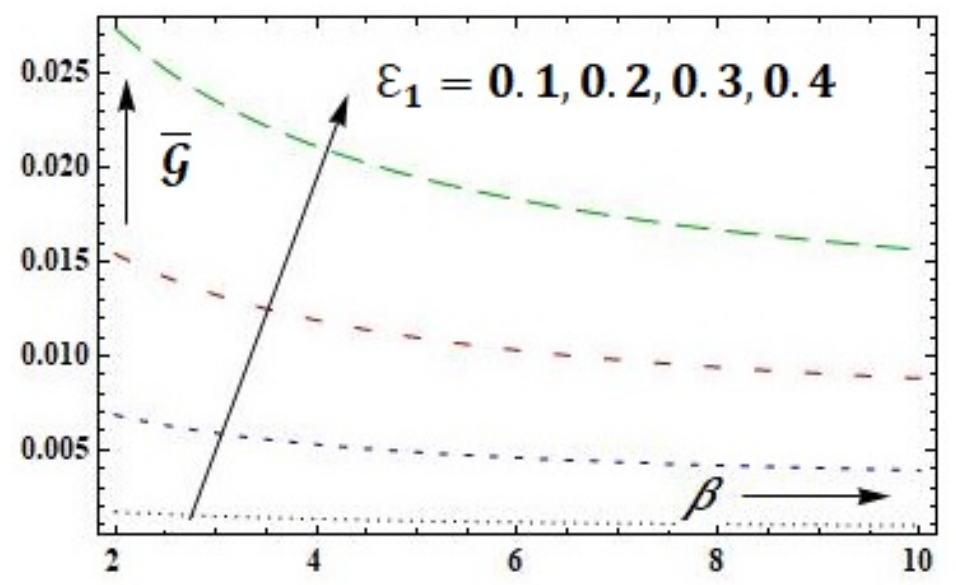

FiguRE 8. Illustration of $\mathcal{E}_{1}$ for $\overline{\mathcal{G}}$ at $\mathcal{D}_{a}=0.9, \mathcal{E}_{2}=0.0, \mathcal{E}_{3}=0.00, \epsilon=0.2, \alpha=1.0, \gamma=0.08$

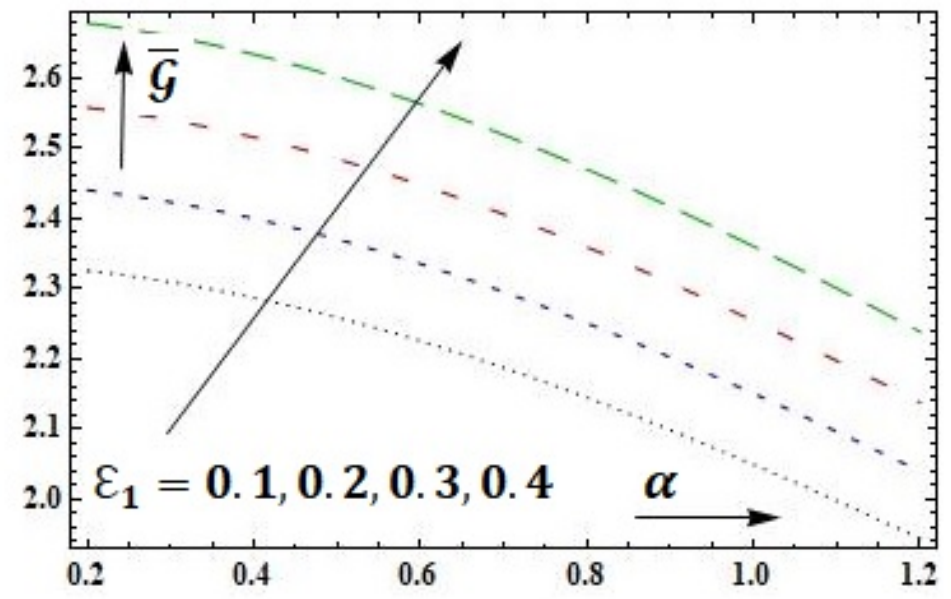

Figure 9. Illustration of $\mathcal{E}_{1}$ for $\overline{\mathcal{G}}$ at $\mathcal{D}_{a}=0.9, \mathcal{E}_{2}=4.0, \mathcal{E}_{3}=0.06, \epsilon=0.2, \beta=5.0, \gamma=0.08$

\section{Conclusions}

In this article, we inspected that the slip and wall effects on two-dimensional creeping flow of a Newtonian fluid through a pervious medium in the existence of chemicals responses. Identical behavior is noticed for wall features, slip constant $\gamma$ and Darcy number $D_{a}$ on dispersion coefficient $(\overline{\mathcal{G}})$ and it is also witnessed the opposite behavior of homogeneous response rate $\alpha$ and heterogeneous response rate $\beta$ are observed on concentration profile. Lastly, it concludes that wall feature constants, slip constraint, and Darcy constant favor diffusion. This model may help in better understanding of the transport phenomena occurring in the intestine leading to absorption of nutrients and drugs. 


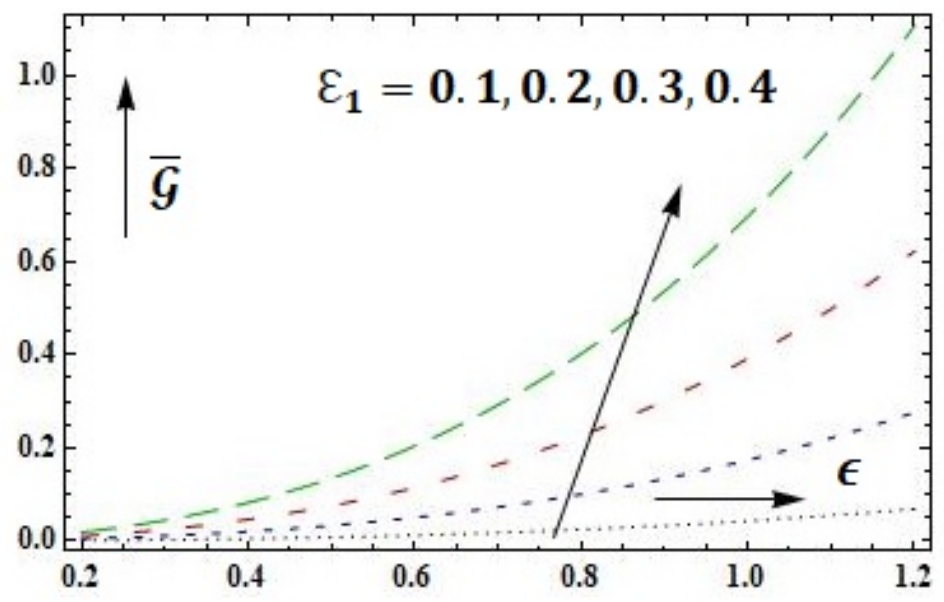

Figure 10. Illustration of $\mathcal{E}_{1}$ for $\overline{\mathcal{G}}$ at $\mathcal{D}_{a}=0.9, \mathcal{E}_{2}=0.0, \mathcal{E}_{3}=0.00, \alpha=1.0, \beta=5.0, \gamma=0.08$

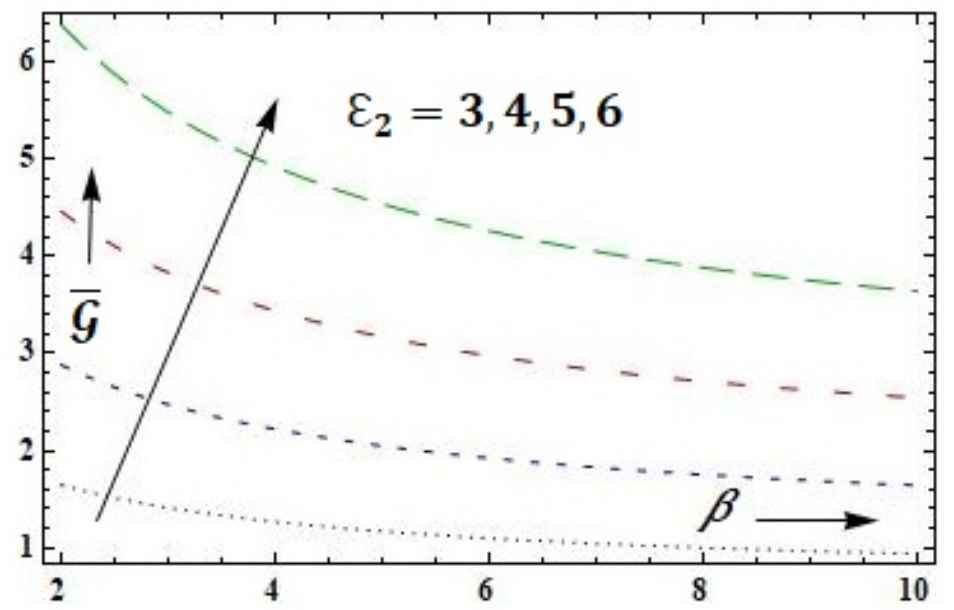

FiguRE 11. Illustration of $\mathcal{E}_{2}$ for $\overline{\mathcal{G}}$ at $\mathcal{D}_{a}=0.9, \mathcal{E}_{1}=0.1, \mathcal{E}_{3}=0.06, \epsilon=0.2, \alpha=1.0, \gamma=0.08$ 


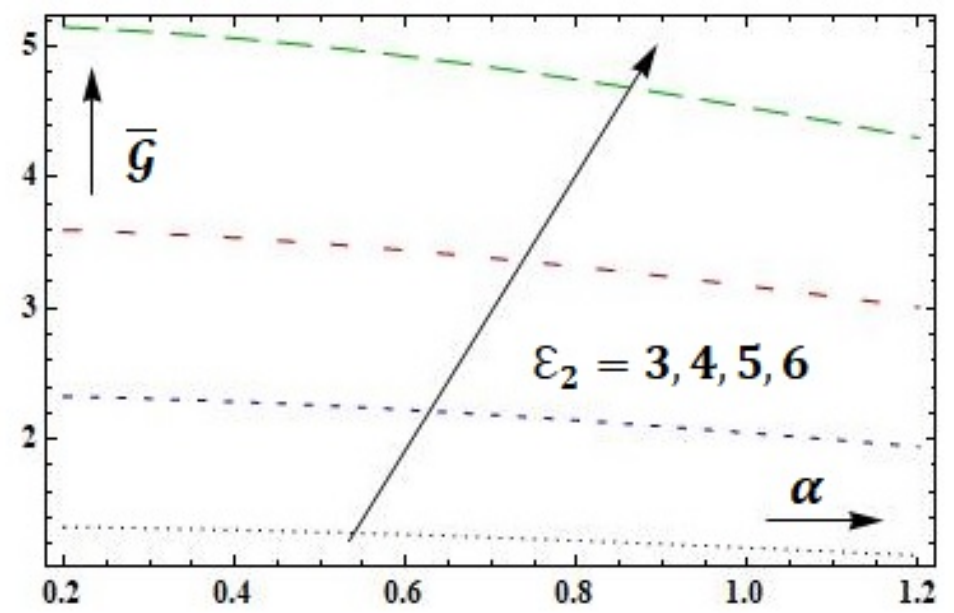

FIgURE 12. Illustration of $\mathcal{E}_{2}$ for $\overline{\mathcal{G}}$ at $\mathcal{D}_{a}=0.9, \mathcal{E}_{1}=0.1, \mathcal{E}_{3}=0.06, \epsilon=0.2, \beta=5.0, \gamma=0.08$

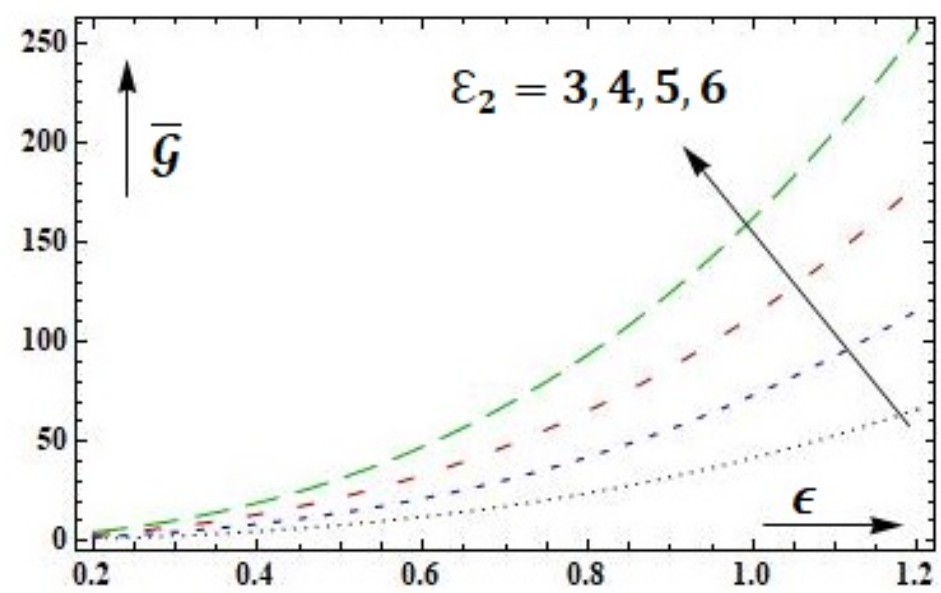

Figure 13. Illustration of $\mathcal{E}_{2}$ for $\overline{\mathcal{G}}$ at $\mathcal{D}_{a}=0.9, \mathcal{E}_{1}=0.1, \mathcal{E}_{3}=0.00, \alpha=1.0, \beta=5.0, \gamma=0.08$ 


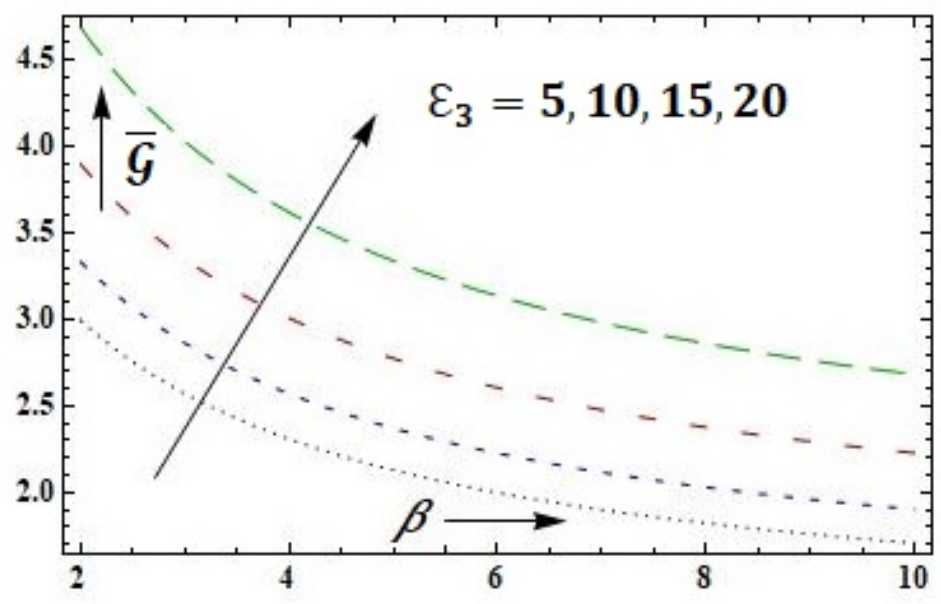

FiguRE 14. Illustration of $\mathcal{E}_{3}$ for $\overline{\mathcal{G}}$ at $\mathcal{D}_{a}=0.9, \mathcal{E}_{1}=0.1, \mathcal{E}_{2}=4.0, \epsilon=0.2, \alpha=1.0, \gamma=0.08$

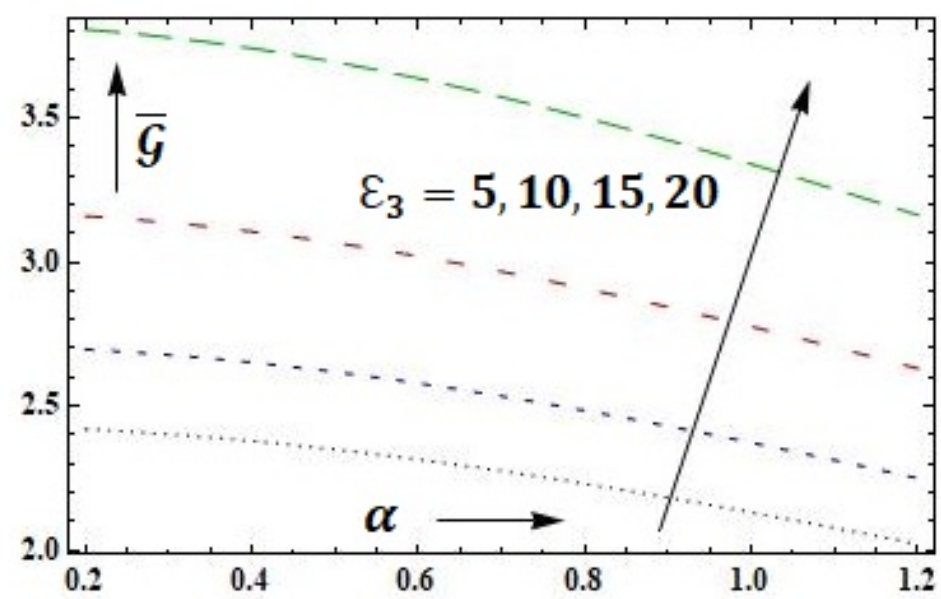

FiguRE 15. Illustration of $\mathcal{E}_{3}$ for $\overline{\mathcal{G}}$ at $\mathcal{D}_{a}=0.9, \mathcal{E}_{1}=0.1, \mathcal{E}_{2}=4.0, \epsilon=0.2, \beta=5.0, \gamma=0.08$ 


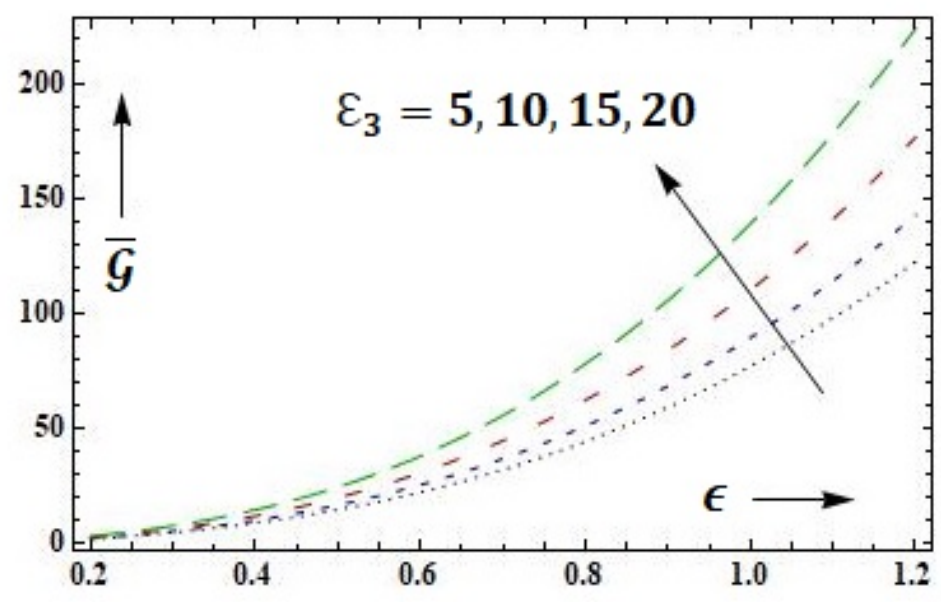

FiguRE 16. Illustration of $\mathcal{E}_{3}$ for $\overline{\mathcal{G}}$ at $\mathcal{D}_{a}=0.9, \mathcal{E}_{1}=0.1, \mathcal{E}_{2}=4.0, \alpha=1.0, \beta=5.0, \gamma=0.08$ 


\section{REFERENCES}

[1] T. W. Latham, Fluid motions in a peristaltic pump, Masters thesis, Massachusetts Institute of Technology, Cambrige, 1966.

[2] Y. C. Fung and C. S. Yih, Peristaltic transport, ASME Transactions: J. Appl. Mech. 35(4)(1968), 669-675.

[3] M. Y. Jaffrin, A. H. Shapiro and S. L. Weinberg, Peristaltic pumping with long wavelengths at low Reynolds number, J. Fluid Mech. 37(1969), 799-825.

[4] D. Takagi and N. J. Balmforth, Peristaltic pumping of viscous fluid in an elastic tube, J. Fluid Mech. 672(2011), 196-218.

[5] T. K. Mittra and N. S. Prasad, On the influence of wall properties and poiseuille flow in Peristalsis, J. Biomechan. 6(1973), 681-693.

[6] A. V. Ramana Kumari and G. Radhakrishnamacharya, Effect of slip and magnetic field on peristaltic flow in an inclined channel with wall effects, Int. J. Biomath. 5(6)(2012), 1250015.

[7] G. C. Sankad and G. Radhakrishnamacharya, Effect of magnetic field on peristaltic motion of micropolar fluid with wall effects, J. Appl. Math. Fluid Mech. 1(2009), 37-50.

[8] G. I. Taylor, Dispersion of soluble matter in solvent flowing slowly through a tube, Proc. Royal Soc. Lond. 219(A)(1953), 186-203.

[9] D. Padma and V. V. RamanaRao, Effect of homogeneous and heterogeneous reactions on the dispersion of a solute in laminar flow between two parallel porous plates, Indian J. Technol. 14(1976), 410-412.

[10] P. S. Gupta and A. S. Gupta, Effect of homogeneous and heterogeneous reactions on the dispersion of a solute in the laminar flow between two plates, Proc. Royal Soc. Lond. 330(A)(1972), 59-63.

[11] P. Chandra and R. P. Agarwal, Dispersion in simple microwfluid flows, Int. J. Eng. Sci. 21(1983), 431-442.

[12] D. Philip and P. Chandra, Effect of heterogeneous and homogeneous reactions on the dispersion of a solute in simple microwfluid, Indian J. Pure Appl. Math. 24(1993), 551-561.

[13] H. Alemayehu and G. Radhakrishnamacharya, Dispersion of solute in peristaltic motion of a couple stress fluid through a porous medium, Tamkang J. Math. 43(4)(2012), 541-555.

[14] G. Ravikiran and G. Radhakrishnamacharya, Effect of homogeneous and heterogeneous chemical reactions on peristaltic transport of a Jeffrey fluid through a porous medium with slip condition, J. Appl. Fluid Mech. 8(3)(2015), 521-528.

[15] A. M. Sobh, Effect of homogeneous and heterogeneous reactions on the dispersion of a solute in MHD Newtonian fluid in an asymmetric channel with peristalsis, Br. J. Math. Computer Sci. 3(4)(2013), 664-679.

[16] K. N. Mehta and M. C. Tiwari, Dispersion in presence of slip and chemical reactions in porous wall tube flow, Defence Sci. J. 38(1988), 1-11.

[17] G. Sankad and M. Dhange, Peristaltic pumping of an incompressible viscous fluid in a porous medium with wall effects and chemical reactions, Alexandria Eng. J. 55(2016), 2015-2021.

[18] D. Pal, Effect of chemical reaction on the dispersion of a solute in a porous medium, Appl. Math. Model. 23(7)(1999), $557-566$.

[19] J. C. Misra and S. K. Ghosh, A mathematical model for the study of blood flow through a channel with permeable walls, Acta Mech. 122(1997), 137-153.

[20] G. S. Beaver and D. D. Joseph, Boundary conditions at a naturally permeable wall, J. Fluid Mech. 30(1967), 197-207.

[21] P. G. Saffman, On the boundary conditions at the surface of a porous medium, Stud. Appl. Math. 1(1971), 93-101.

[22] B. S. Bhatt and N. C. Sacheti, On the analogy in slip flows, Indian J. Pure Appl. Math. 10(1979), 303-306. 